\title{
what's your diagnosis?
}

\section{Diagnosis: Multiple endocrine neoplasia type 1 (MEN 1), also known as Wermer's syndrome}

\author{
Chandan Jyoti Das, ${ }^{*}$ Jyotindu Debnath,* NG Javan, $†$ Manash Pratim Baruah $\neq$ \\ From the Departments of *Radiology, +Surgery and \#Endocrinology, All India Institute of Medical Sciences, New Delhi, India
}

Correspondence and reprint requests: Dr. Chandan Jyoti Das - All India Institute of Medical Sciences - Radiology • New Delhi 29, India New Delhi 110029, India · T: +91-11-2658500 · F: +91-11-26588663 - Accepted for publication March 2007

Ann Saudi Med 2007; (27)5: 388-389

$\mathrm{M}$ $\mathrm{RI}$ revealed a soft tissue mass in the sella causing its expansion. The mass was hypointense on T1W images (Figure1a) showing peripheral enhancement on post gadolinium image (Figure 1b). A plain radiograph of the hands shows osteopenia with subperiosteal erosion in the phalanges (Figure 2). A diagnosis of pituitary macroadenoma was suggested based on the MR appearance. She was put on bromocriptine therapy following which her periods became regular. In view of subperiosteal erosion seen on the plain radiograph, a suspicion of hyperparathyroidism was made as subperiosteal erosion of the bone, particularly along the radial aspect of the middle phalanx of middle and index finger, which is virtually pathognomonic for hyperparathyroidism although osteopenia can be found in other conditions, viz. osteomalacia, multiple myeloma. Contrast enhanced CT scan of neck was performed, which revealed enlarged right inferior parathyroid gland. MRI of the abdomen was also performed which revealed a small well defined space occupying lesion in the proximal body of pancreas, which was uniformly hypointense on T1W image and hyperintense on T2W image (Figure 3a, 3b). Bilateral adrenal hyperplasia was also noted. Based on the clinical profile, laboratory findings and imaging appearance, a diagnosis of MEN 1 syndrome was suggested. She underwent trans-sphenoidal decompression of the mass and histopathology confirmed the diagnosis of pituitary adenoma. The patient also underwent total parathyroidectomy, enucleation of the pancreatic tumor. Histopathology of the resected specimens showed parathyroid hyperplasia and neuroendocrine tumor of the pancreas. She is on clinical follow up and is doing well.

\section{DISCUSSION}

Multiple endocrine neoplasia type 1 (MEN 1) also known as Wermer's syndrome, is an autosomal dominant genetic syndrome characterized by a predisposition of simultaneous or successive development of neoplasia

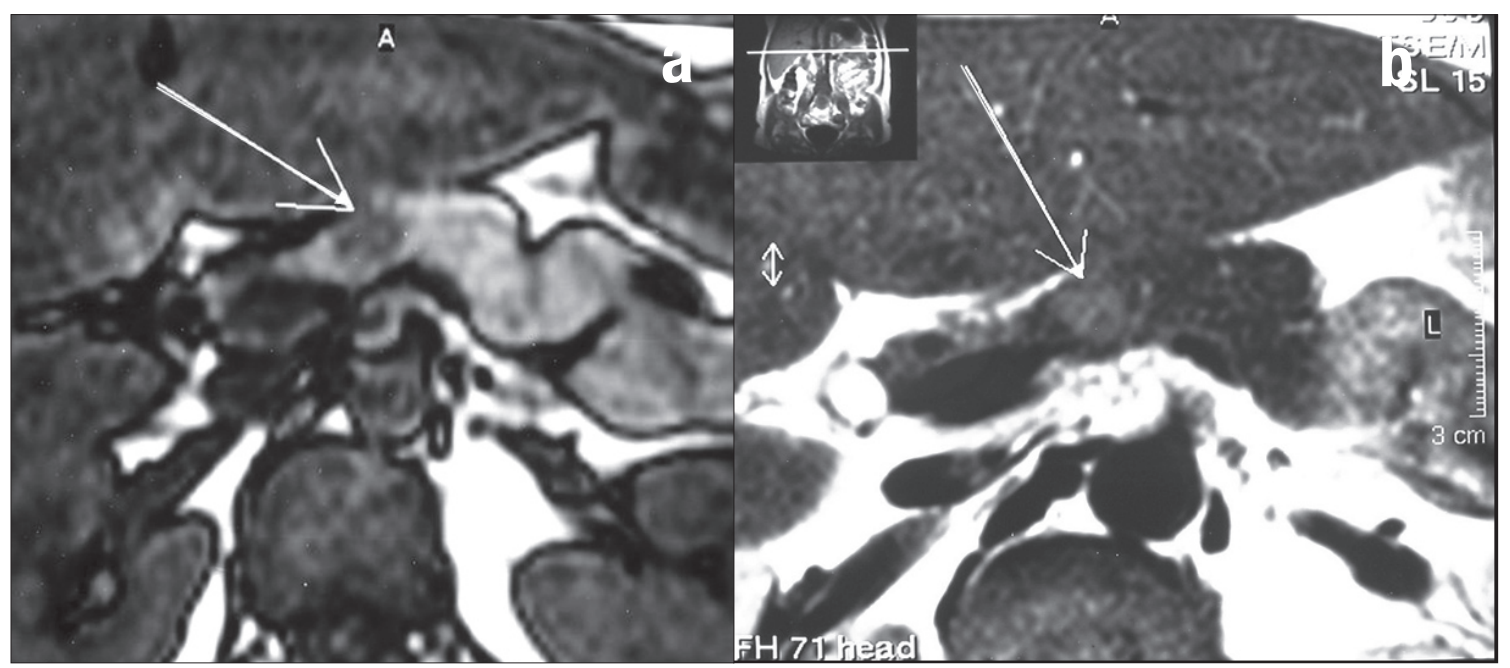

Figure 3. T1 weighted axial MR image showing a hypointense lesion in the proximal body of the pancreas (arrow) (a).The lesion appears mildly hyperintense on TSE T2 weighted axial image (arrow) (b). 
and or hyperplasia of several endocrine glands. ${ }^{1}$ It is caused by a germ line mutation in the MEN 1 gene locus coding for a tumor suppressor protein, called menin. ${ }^{2}$ Jacob Erdheim in 1903 first described this condition in a patient with tumors involving two endocrine organs, the pituitary gland and the parathyroid glands and almost six decades later Steiner et al introduced the term "Multiple Endocrine Neoplasia" to describe a combination of tumors of three different types of endocrine glands viz. familial parathyroid, pituitary and pancreatic islet cell tumors (MEN type 1), familial pheochromocytoma, medullary thyroid carcinoma and hyperparathyroidism (MEN type 2) and non familial parathyroid tumors and papillary thyroid carcinomas (MEN type 3$){ }^{3}$ Classical MEN 1 includes primary hyperparathyroidism (HPT), enteropancreatic neuroendocrine tumors (EPNET) and pituitary adenomas. ${ }^{4}$

Primary hyperparathyroidism is the most common manifestation of MEN 1 syndrome and most of the affected individuals develop hypercalcemia by age 40 . Parathyroid hyperplasia is the most common cause of hyperparathyroidism in MEN 1, although single and multiple adenomas have been described. ${ }^{5}$ Manifestations of hyperparathyroidism in MEN 1 includes nephrolithiasis, pathological fractures, gastrointestinal and musculoskeletal complaints. Enteropancreatic neuroendocrine tumors (EPNET) are the second most common manifestation of MEN $1 .{ }^{1}$ Most frequent tumors are gastrinomas and insulinomas. ${ }^{1}$ Early identification of EPNET is of critical importance as these tumors represent the most frequent cause of death with this syndrome. ${ }^{2}$ Pituitary tumors occur in more than half of patients with MEN 1 and tend to be multicentric, making them difficult to resect. Prolactinomas are the most common and are diagnosed by finding serum prolactin levels $>200 \mu \mathrm{g} / \mathrm{L}$ and can be picked up on dynamic CEMRI. ${ }^{5}$ Clinical symptom depends on the size of the adenoma and the intensity of hormone secretion. In addition, MEN 1 carriers can have various other endocrine or non endocrine lesions like adrenal hyperplasia, thymoma, thymic and foregut carcinoids ${ }^{3}$
The diagnosis of MEN 1 syndrome is established by family screening, clinical data, and appropriate hormonal assay and stimulation tests. Family history of similar disease is very important as first-degree relatives of MEN 1 patient have about $50 \%$ risk of development of the disease. ${ }^{6}$ Imaging studies play a crucial role in identification of the target organs of lesions suspected on the basis of clinical and laboratory findings, staging of malignant lesions and search for an occult co-existing lesion to enable correct medical and or surgical treatment planning. For parathyroid lesion, high resolution ultrasonography (US) of neck preferably with a linear probe is the initial imaging modality of choice. If neck US is negative, CT, MRI or nuclear scintigraphy is indicated for demonstration of ectopic location of parathyroid in the anterior mediastinum. ${ }^{1}$ Currently MRI is the technique of choice for evaluation of the pituitary tumors in MEN 1 patients. ${ }^{1}$ Abdominal USG has a sensitivity of about $60 \%$ for insulinoma, and $20-30 \%$ for gastrinoma. Endoscopic ultrasonography (EUS) has improved the rate of detection of tumors in the pancreas and duodenal wall. Intraoperative USG combined with surgical palpation is another reliable method for detection of EPNET. ${ }^{1}$ CT has overall sensitivity of $50 \%$ for the detection of pancreatic endocrine tumors. In addition to locoregional staging, USG and CT are particularly useful for detection of liver metastases, which is crucial before any definite therapeutic planning. ${ }^{1}$ Somatostatin receptor scintigraphy and selective intra-arterial stimulation testing with secretin have been found to have greatest sensitivity in localizing gastrinoma. ${ }^{1}$ MRI has an important role in identification of EPNET. The use of fast spin echo (FSE) and fat saturation techniques are particularly useful in imaging pancreatic lesion especially islet cell tumor. ${ }^{7}$

Genetic screening in first degree relatives of MEN1 patients may be advisable during early adolescence to assess risk of developing MEN1 in future. In symptomatic individuals of MEN1, annual clinical and biochemical (calcium, PTH, gastrin, prolactin) follow-up as well as routine pancreatic and pituitary imaging may be complemented on individual merit basis.

\section{REFERENCES}

1. Padovani $B$, Chevallier P. Multiple endocrine neoplasia syndrome. In: Bruneton JN editor. Radiological imaging of endocrine diseases. 1st ed. Berlin - New York: Springer, 1999; 257-63. 2. Doherty GM. Multiple endocrine neoplasia type 1. J Surg Oncol 2005; 89:143-50.

3. Carney JA. Familial multiple endocrine neoplasia - the first 100 years. Am J Surg Pathol
2005; 29:254-74.

4. Chanson P, Cadiot G, Murat A. Management of patients and subjects at risk for multiple endocrine neoplasia type 1: Horm Res 1997;47:211-

5. Sherman SI, Gagel RF. Disorders affecting multiple endocrine systems. In: Kasper DL editor. Harrison's Principles of Internal Medicine. 16st ed. McGraw-Hill, 2005; 2231-38.

6. Trump D, Farren B, Wooding $C$ et al. Clinical studies of multiple endocrine neoplasia type 1 (MEN1). QJM. 1996;89:653-69.

7. Owen NJ, Sohaib SAA, Peppercorn PD, Monson JP, Grossman AB, Besser GM et al. MRI of pancreatic neuroendocrine tumors. Br J Radiol.2001; 74:968-73. 